Norris, J. R. (1957). J. gen. Microbiol. 16, 1-8

\title{
A Bacteriolytic Principle Associated with Cultures of Bacillus cereus
}

\author{
BY J. R. NORRIS \\ Department of Agriculture, University of Leeds
}

SUMMARY : A lytic principle associated with cultures of Bacillus cereus is described and some of its properties discussed. The principle is relatively non-specific, acting on several species of aerobic spore-formers. It has properties in common with similar agents reported recently and comparisons with these are made. Lysis results in the loss of cell contents whilst at least part of the cell-wall structure remains intact.

Delpy \& Chamsy (1949) found that in suspensions of old cultures of Bacillus anthracis, incubated in the presence of $1 / 20,000$ thiomersalate, the vegetative rods underwent lysis which was complete in 4 days, the spores retaining their viability and serological properties. It is an extension of this observation that has led to the demonstration of a bacteriolytic principle associated with cultures of $\boldsymbol{B}$. cereus.

\section{METHODS}

Organisms. The strain which produces the lytic principle used in this work (Bacillus cereus M. 8) was originally isolated from Egyptian soil (Mahmoud, 1955). It satisfied the main diagnostic criteria of Smith, Gordon \& Clark (1952) and was strongly lecithinase-positive. It has been maintained by subculture on nutrient agar at $30^{\circ}$. The other organisms used for testing the principle were $B$. subtilis NCTC 3610 and the following strains from the Wellcome Research Laboratories: B. circulans 2925, B. sphaericus 1652, B. polymyxa 2002, B. alvei 2198 and B. brevis 2934 (Knight \& Proom, 1950).

Thiomersalate. The preparation used was a $1 / 1000$ solution of sodium mercurithiosalicylate obtained from Eli Lilley and Co. Ltd., Basingstoke.

Filter membranes. Courtauld's cellulose acetate membranes were used.

Culture media. Cultures were grown on Oxoid nutrient agar in 20 oz. Roux bottles which were each inoculated with $1 \mathrm{ml}$. of an overnight broth culture and incubated at $30^{\circ}$. For fluid cultures Oxoid nutrient broth was inoculated with a loopful of an overnight broth culture and incubated at $30^{\circ}$, with gentle shaking, in a water bath.

The production of the lytic principle. A Roux bottle containing nutrient agar was inoculated with Bacillus cereus $\mathrm{M} 8$ and incubated for $12 \mathrm{hr}$. at $30^{\circ}$. The growth was then washed off into $10 \mathrm{ml}$. of sterile distilled water and the organisms centrifuged down to give an actively lytic supernatant fluid which could then be treated with thiomersalate or filtered through a sterilizing membrane as required.

The production of a bacterial suspension for use as substrate in the assay of lytic activity. For assaying lytic activity it was essential to use an organism 
which showed no tendency to autolyse when suspended in distilled water (with or without thiomersalate) or in a preparation of the lytic principle which had been inactivated by heat. Most of the species investigated did in fact autolyse to some extent under these conditions, but Bacillus sphaericus 1652, whilst fully susceptible to the lytic principle, was stable in its absence and this organism was used throughout.

Bacillus sphaericus 1652 was grown in nutrient broth for $12-16 \mathrm{hr}$., centrifuged down, washed twice in sterile distilled water and resuspended in sterile distilled water to give a concentration of approximately $4 \times 10^{8}$ organisms $/ \mathrm{ml}$. This concentration, when diluted 10 times, gave a reading of about 100 units on the EEL nephelometer with the instrument set at two-thirds maximum sensitivity.

Method of assay of lytic activity. Samples $(8.5 \mathrm{ml}$.) of the preparations to be tested were placed in sterile rubber-stoppered test tubes; $1 \mathrm{ml}$. of the Bacillus sphaericus 1652 suspension and $0.5 \mathrm{ml}$. of a $1 / 1000$ solution of thiomersalate were then added to give a final thiomersalate concentration of $1 / 20,000$. Each assay was performed in duplicate, the tubes being incubated in a water bath at $37^{\circ}$ and nephelometer readings taken at intervals.

During the lysis of initially viable organisms there was a preliminary increase in nephelometer reading. The extent of this increase tended to differ from test to test and interfered with the comparison of the influence of variables such as temperature on the rate of lysis. When the $B$. sphaericus suspension was allowed to incubate in the presence of $1 / 20,000$ thiomersalate for $3 \mathrm{hr}$. before addition of the lytic agent, no initial increase of optical density took place and a suspension pretreated in this manner was used whenever direct comparisons were to be made. It should be emphasized that such organisms were nonviable, since when washed $B$. sphaericus 1652 was suspended in distilled water there was a rapid decrease in viable count, the rate of which was not affected by the addition of thiomersalate (Fig. 1).

Staining techniques. The Gram method was carried out in the usual way with an acetone/ethanol mixture as decolorizing agent (Kopeloff \& Cohen, 1928). Robinow's tannic acid-crystal violet method (Robinow, 1945) was used to stain cell"walls.

Viable counts. In the absence of thiomersalate viable counts were performed in the usual way, using quarter-strength Ringer's solution as diluent and nutrient agar as the medium. When thiomersalate was present it was inactivated by the incorporation of glutathione to $3 \times 10^{-8} \mathrm{M}$ into the medium.

\section{RESULTS}

\section{Lytic activity and age of culture}

Preliminary experiments showed that the lytic activity associated with fluid cultures of Bacillus cereus $\mathbf{M} 8$ was of a low order compared with that of cultures grown on solid media. A number of nutrient agar cultures was set up in Roux bottles and incubated at $30^{\circ}$. The total growth from a bottle was harvested into $10 \mathrm{ml}$. sterile distilled water at 8,12 and $16 \mathrm{hr}$., the organisms 
centrifuged down and the supernatant fluid treated with thiomersalate and assayed for lytic activity against $B$. sphaericus 1652 . Comparison of the initial rates of decrease in nephelometer reading indicated that there was little difference in the activities of the three preparations (Fig. 2). A $12 \mathrm{hr}$. incubation period was adopted for the preparation of the lytic principle.

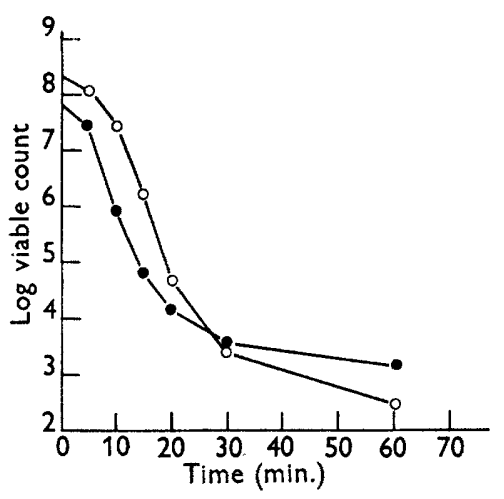

Fig. 1

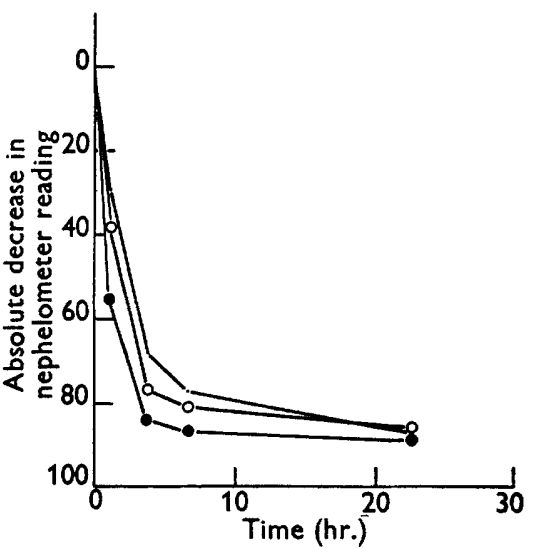

Fig. 2

Fig. 1. The loss of viability of washed vegetative organisms from a $16 \mathrm{hr}$. broth culture of Bacillus sphaericus when suspended in distilled water. In the presence $\mathrm{O}-\mathrm{O}$ and absence $1 / 20,000$ thiomersalate.

Fig. 2. A comparison of the rates of lysis of vegetative organisms of Bacillus sphaericus suspended in supernatant fluids derived from cultures of $B$. cereus in the presence of thiomersalate. $B$. cereus cultures harvested at $-12 \mathrm{hr}$., $12 \mathrm{hr}$. and $\mathrm{O}-\mathrm{O} 16 \mathrm{hr}$.

Since Ivanovics \& Alfoldi (1955) found that their agent, Megacine, was released during the lysis of the organism which produced it, the deposit from the $12 \mathrm{hr}$. culture of Bacillus cereus M8 in the above experiment was washed and resuspended in sterile distilled water to which thiomersalate was added. Autolysis, as judged by nephelometer reading and microscopically, occurred at $37^{\circ}$ over a period of $24 \mathrm{hr}$. and samples of supernatant fluid taken at intervals were assayed for lytic acitivity against $B$. sphaericus 1652 . There was no significant increase in lytic activity accompanying autolysis.

\section{Properties of the lytic principle}

Filterability. There was only slight loss of activity on passage through a membrane filter.

Optimum temperature for activity. Replicate assays were set up in water baths at different temperatures and the rates of lysis compared (Fig. 3); activity was optimal at $37^{\circ}$. Controls consisting of suspensions of Bacillus sphaericus 1652 in heat-inactivated preparations of the lytic principle, and similar suspensions in distilled water, both containing $1 / 20,000$ thiomersalate, were set up at all the different temperatures and showed no significant change in optical density during the course of the experiment. 
Dialysability. A preparation was dialysed in a cellophan bag against running water at $4^{\circ}$. There was no significant loss of activity at $20 \mathrm{hr}$. and the agent appeared to be non-dialysable.

Thermal stability. Samples of an active preparation were heated for $\mathbf{3 0}$ min. at different temperatures in water baths and their activities compared (Fig. 4). Loss of activity was almost complete following exposure to $70^{\circ}$ for $30 \mathrm{~min}$., and complete at $80^{\circ}$ for the same length of time.

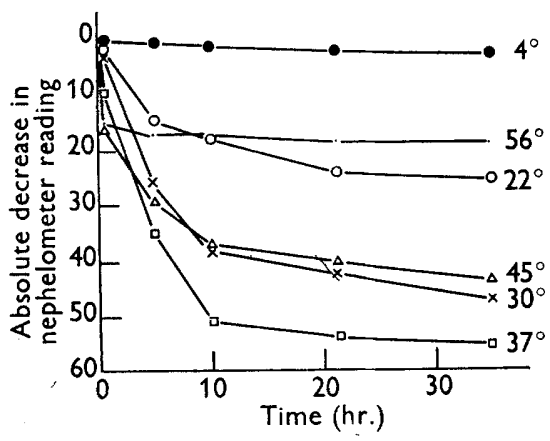

Fig. 3

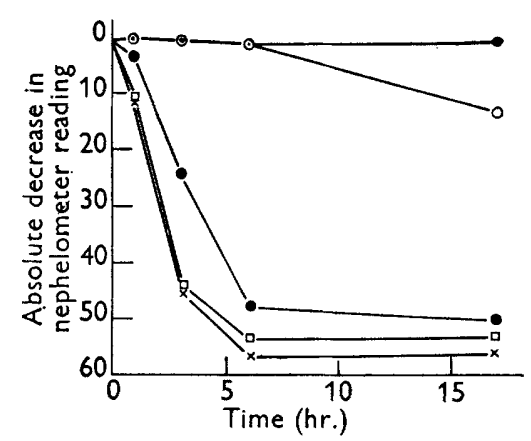

Fig. 4

Fig. 3. The effect of temperature on the rate of lysis of vegetative Bacillus sphaericus suspended in a preparation of the $B$. cereus lytic principle $+1 / 20,000$ thiomersalate.

Fig. 4. The thermal stability of the Bacillus cereus lytic principle. Vegetative B. sphaericus was suspended in preparations of the principle in the presence of thiomersalate. Preparation unheated $\square-\square$, and heated for $30 \mathrm{~min}$. at $\times \longrightarrow 5^{\circ}$, $0 \mathrm{r0}^{\circ}$ and $-\longrightarrow 80^{\circ}$.

Optimum $\mathrm{pH}$ value. The normal $\mathrm{pH}$ value of the preparation was $\mathrm{pH} 8 \cdot \mathbf{0}-\mathbf{8} \cdot \mathbf{2}$ and adjustment of reaction to the acid side resulted in considerable precipitation of material which interfered with the use of the nephelometer so that it was not possible to survey the activity at a range of $\mathrm{pH}$ values.

Specificity. The agent was tested against strains of the following members of the genus Bacillus and lysed all of them: B. cereus, B. circulans, B. polymyxa, B. subtilis, B. alvei, B. brevis, B. sphaericus. There was no lysis of Staphylococcus aureus (Oxford strain) or a strain of Escherichia coli.

Lecithinase and gelatinase activities. Complex preparations derived from a strongly proteolytic organism such as Bacillus cereus, may be expected to possess a variety of enzymic properties, and tests for lecithinase and gelatinase activities were both strongly positive. What part, if any, such activities play in the lytic process remains to be shown.

Action on heat-killed organisms. Vegetative Bacillus sphaericus 1652, killed by heating at $80^{\circ}$ for $20 \mathrm{~min}$., was rapidly lysed by preparations of the principle. Lysis of such organisms was not preceded by an increase in turbidity of the suspension as was noted earlier with unheated organisms (Fig. 5).

Cytological changes during lysis. Examined by the Gram method, lysis of Bacillus sphaericus 1652 appeared to consist of two distinct stages. The initial decrease in turbidity was associated with a loss of Gram-positive reaction. The rods, initially uniformly Gram-positive, rapidly became granular and the 
Gram-positive granules then became fewer in number and stained less intensely. At the end of this phase the organisms stained well with the counterstain. During the second phase staining became fainter until the organisms appeared as barely visible ghosts; finally only debris was seen. When such a lysed preparation was stained with tannic acid-crystal violet, however, the cell walls were seen to be intact although almost devoid of contents, an observation confirmed by electron microscopy (Pl. 2, fig. 6). The details of lysis were essentially similar with all the seven organisms studied. Control suspensions of vegetative organisms in heat-inactivated lytic principle showed none of these changes during the course of the experiment (Pl. 2, fig. 5).

Bactericidal activity. The bactericidal activity of sterile (membrane filtered) thiomersalate-free preparations of the principle was investigated by adding $1 \mathrm{ml}$. of a $16 \mathrm{hr}$. broth culture of test organism to $9 \mathrm{ml}$. of the lytic preparation and following changes in viable count over a period of $15 \mathrm{hr}$. Controls consisted of similar inocula added to heat-inactivated material. The preparations showed a marked bactericidal activity against Bacillus cereus, B. subtilis and Staphylococcus aureus but did not affect Bacillus sphaericus, B. circulans or Escherichia coli. Death did not occur in the controls, and in most cases active growth took place (Table 1).

Table 1. The bactericidal activity of the lytic principle from Bacillus cereus $M 8$

$1 \mathrm{ml}$. of an overnight broth culture of each test organism was added to $9 \mathrm{ml}$. of filtered lytic principle preparation, and the changes in viable count followed during incubation at $\mathbf{3} 7^{\circ}$.

\begin{tabular}{|c|c|c|c|c|c|}
\hline \multicolumn{3}{|c|}{$\begin{array}{l}\text { Organisms suspended in solution } \\
\text { containing lytic principle }\end{array}$} & \multicolumn{3}{|c|}{$\begin{array}{l}\text { Organisms suspended in solution } \\
\text { containing lytic principle inactivated } \\
\text { by heating at } 80^{\circ} \text { for } 20 \text { min. }\end{array}$} \\
\hline \multicolumn{6}{|c|}{ Time of observation (hr.) } \\
\hline 0 & 5 & 16 & 0 & 5 & 16 \\
\hline \multicolumn{6}{|c|}{ Viable counts (organisms $/ \mathrm{ml}$.) } \\
\hline $235 \times 10^{3}$ & $24 \times 10^{2}$ & $162 \times 10^{1}$ & $219 \times 10^{3}$ & $51 \times 10^{4}$ & $30 \times 10^{5}$ \\
\hline $42 \times 10^{4}$ & $16 \times 10^{4}$ & $89 \times 10^{2}$ & $38 \times 10^{4}$ & $42 \times 10^{4}$ & $95 \times 10^{5}$ \\
\hline $71 \times 10^{4}$ & $9 \times 10^{1}$ & $18 \times 10^{0}$ & $77 \times 10^{4}$ & $23 \times 10^{4}$ & $99 \times 10^{4}$ \\
\hline $140 \times 10^{4}$ & $26 \times 10^{5}$ & $162 \times 10^{6}$ & $131 \times 10^{4}$ & $22 \times 10^{5}$ & $22 \times 10^{6}$ \\
\hline $52 \times 10^{4}$ & $127 \times 10^{3}$ & $187 \times 10^{4}$ & $46 \times 10^{4}$ & $59 \times 10^{4}$ & $48 \times 10^{5}$ \\
\hline $57 \times 10^{5}$ & $65 \times 10^{5}$ & $46 \times 10^{6}$ & $52 \times 10^{5}$ & $74 \times 10^{5}$ & $57 \times 10^{6}$ \\
\hline
\end{tabular}

The role of thiomersalate in the lytic process. Reference to Figs. 4-6 shows that in each of the control preparations vegetative Bacillus sphaericus 1652 showed no tendency to lyse in the absence of active lytic principle, and it may be concluded that thiomersalate had no lytic activity of its own under the conditions described. Thiomersalate proved to be essential for the initial increase in turbidity of the suspensions of $B$. sphaericus 1652 which were undergoing lysis, and its presence greatly facilitated the action of the lytic principle (Fig. 6). 


\section{DISCUSSION}

Early reports of bacteriolytic activity associated with spore-forming aerobic bacteria are numerous (Waksman, 1945). Recently attention has been redirected to this subject by workers who have paid more attention to characterizing the agents concerned. Ivanovics \& Alfoldi (1955) induced rapid lysis in cultures of certain strains of Bacillus megaterium by ultraviolet irradiation and found that the resulting lysate was very active in promoting the lysis of all

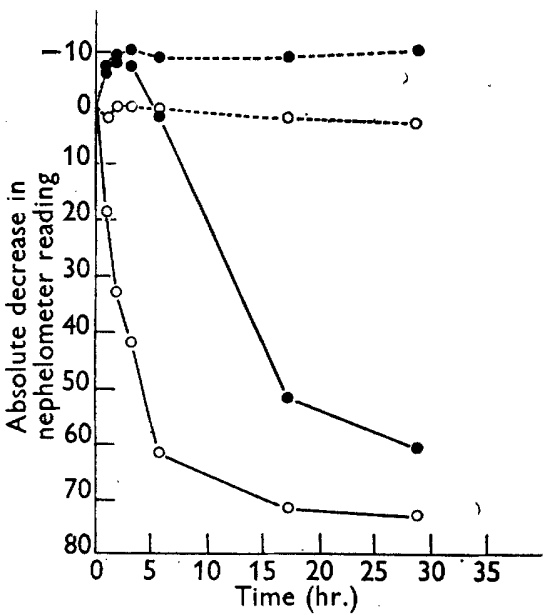

Fig. 5

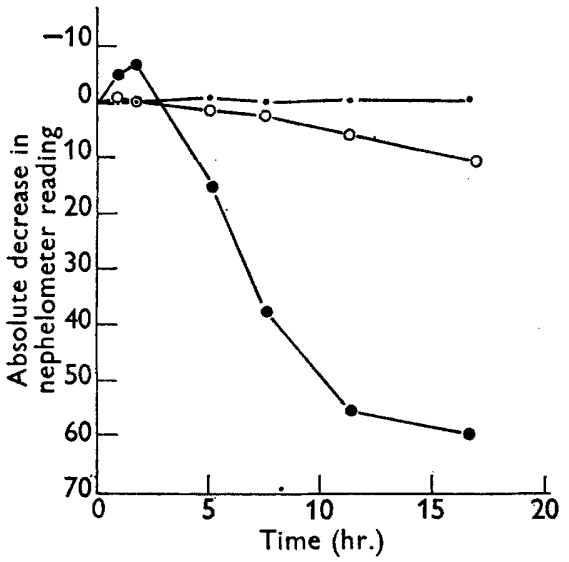

Fig. 6

Fig. 5. The action of the Bacillus cereus lytic principle on heated and unheated vegetative $B$. sphaericus. Unheated organisms suspended in lytic principle, and in heatinactivated principle - - O. Organisms heated at $80^{\circ}$ for $20 \mathrm{~min}$. and suspended in lytic principle $\bigcirc-O$, and in heat-inactivated principle $\bigcirc-\cdots$.

Fig. 6. Vegetative Bacillus sphaericus suspended in lytic principle with $1 / 20,000$ thiomersalate, and $\mathrm{O}-\mathrm{O}$ without thiomersalate. - - Organisms suspended in heat-inactivated lytic principle + thiomersalate.

strains of this organism tested. The agent was released during the lysis of the organism which produced it, was bactericidal and had properties indicative of a protein-like nature; it was largely species-specific. Greenberg \& Halvorson (1955) demonstrated a lytic agent in old fluid cultures of $B$.terminalis (B. cereus). This agent was also largely species-specific, attacking only $\boldsymbol{B}$. cereus strains, and had protein-like properties. Similar agents were demonstrated in two strains of $\boldsymbol{B}$. cereus and B. polymyxa. Neither of these papers described the cytological changes which accompanied lysis. Boroff (1955) with the anaerobe Clostridium botulinum demonstrated in old cultures a lytic agent which was active against young actively growing cultures of the same organism. Lysis was followed microscopically with Gram-stained organisms; the series of changes seen closely resembled those described in the present paper. Cell-wall stains were not used, however, and the presence of cell-wall material at the end of lysis in these experiments must remain problematical. Boroff's agent was not characterized but was destroyed by boiling for $5 \mathrm{~min}$. 
The lytic principle described in the present paper resembles those investigated by the above workers in its main properties, which are consistent with its being protein in nature. The conditions under which it is formed and its marked non-specificity, however, serve to distinguish it from them. One cannot put forward more than tentative suggestions as to the mode of action of the agent. The nature of the lysis, the marked non-specificity and the ability to act on non-viable organisms speak against a phage mechanism, a conclusion supported by failure to demonstrate any increase in lytic activity during lysis of the substrate organism. Lysis might well be the result of the combined action of more than one constituent of the preparation, one possibly acting on the cell walls in such a way as to facilitate the entry of an actively lytic component into the cell. Concerning the role of thiomersalate, at the concentration used it causes little protein denaturation (McCulloch, 1945) and its activity in facilitating the action of the lytic principle may be attributable to interference with the normal metabolic processes of the organism, resulting in an enhanced susceptibility to the agent. Greenberg \& Halvorson (1955) obtained a correlation between decrease in viable count and decrease in turbidity, attributing both effects to the action of their lytic agent. It should be borne in mind that preparations from organisms of this type may well possess additional antibiotic substances (Snell, Ijichi \& Lewis, 1956) and a direct correlation between lysis and bactericidal activity need not necessarily exist. In the case of the agent here described the bactericidal action against Staphylococcus aureus was not associated with lysis; the two effects must be distinguished. In addition to their more general applications these observations serve to emphasize that decrease in optical density, which has often been used as an indicator of lysis, is a criterion which must be interpreted with caution since, although the contents of the cells are apparently going into solution, it does not necessarily follow that the cell walls are being disintegrated.

The author is indebted to Mr J. Wolf, Head of the Bacteriology Section for advice and criticism and to Mr G. W. Ripley of the Botany Department for the electron micrographs. The work was carried out during the tenure of an Agricultural Research Council Research Scholarship.

\section{REFERENCES}

Boroff, D. A. (1955). Studies of toxins of Clostridium botulinum. III. Relation of autolysis to toxin production. J. Bact. 70, 363.

Delpy, P. L. \& Chamsy, H. M. (1949). Sur la stabilisation des suspensions sporulées de B. anthracis parl'action des certains antiseptiques. C.R. Acad.Sci., Paris, 228, 1071.

Greenberg, R. A. \& Halvorson, H. O. (1955). Studies on an autolytic substance produced by an aerobic spore forming bacterium. J. Bact. 69, 45 .

Ivanovics, G. \& ALFoldi, L. (1955). Observations on lysogenesis in B. megaterium and on megacine, the antibacterial principle of this bacillus species. Acta Microbiologica, 2, 275.

KNight, B. C. J. G. \& Proom, H. (1950). A comparative survey of the nutrition and physiology of mesophilic species in the genus Bacillus. J. gen. Microbiol. 4, 508.

Kopeloff, N. \& Cohen, P. (1928). Further studies on a modification of the Gram stain. Stain Tech. 3, 64. 
McCulloch, E. C. (1945). Disinfection and Sterilization, 2nd ed. p. 272. London: H. Kimpton.

Mahmoud, S. A. Z. (1955). A Study of Sporeformers Occurring in Soils. Their Germination and Biochemical Activity. Ph.D. Thesis, University of Leeds.

Robinow, C. F. (1945). Addendum to Dubos, The Bacterial Cell, p. 372. Harvard: University Press.

Smith, N. R., Gordon, R. E. \& Clakk, F. E. (1952). Aerobic Spore-forming Bacteria. Washington, U.S. Department of Agriculture, Monograph. no. 16.

Snell, N., IJICHI, K. \& Lewis, J. C. (1956). Paper chromatographic identification of polypeptide Gram-positive inhibiting antibiotics. Appl. Microbiol. 4, 13.

Waksman, S. A. (1945). Microbial Antagonisms and Antibiotics. New York: The Commonwealth Fund.

\section{EXPLANATION OF PLATES}

Plate 1

Changes in the appearance of vegetative Bacillus sphaericus 1652 suspended in solution containing the lytic principle and 1/20,000 thiomersalate; incubated at $37^{\circ}$. Light microscope; $\times 1250$ approximately.

Fig. 1. Organisms at the beginning of lysis. Gram stain.

Fig. 2. Organisms after $2 \mathrm{hr}$. of lysis. Gram stain.

Fig. 3. Debris left at the end of $16 \mathrm{hr}$. of lysis. Gram stain.

Fig. 4. Washed deposit from the preparation in fig. 3. Cell-wall stain.

\section{Plate 2}

Electron micrographs of vegetative Bacillus sphaericus 1652.

Fig. 5. Organisms suspended in heat-inactivated lytic principle $+1 / 20,000$ thiomersalate and incubated for $16 \mathrm{hr}$. $\times 4300$.

Fig. 6. Organism suspended in active lytic principle $+1 / 20,000$ thiomersalate and incubated for $16 \mathrm{hr}$. $\times 21,500$.

(Received 8 May 1956) 


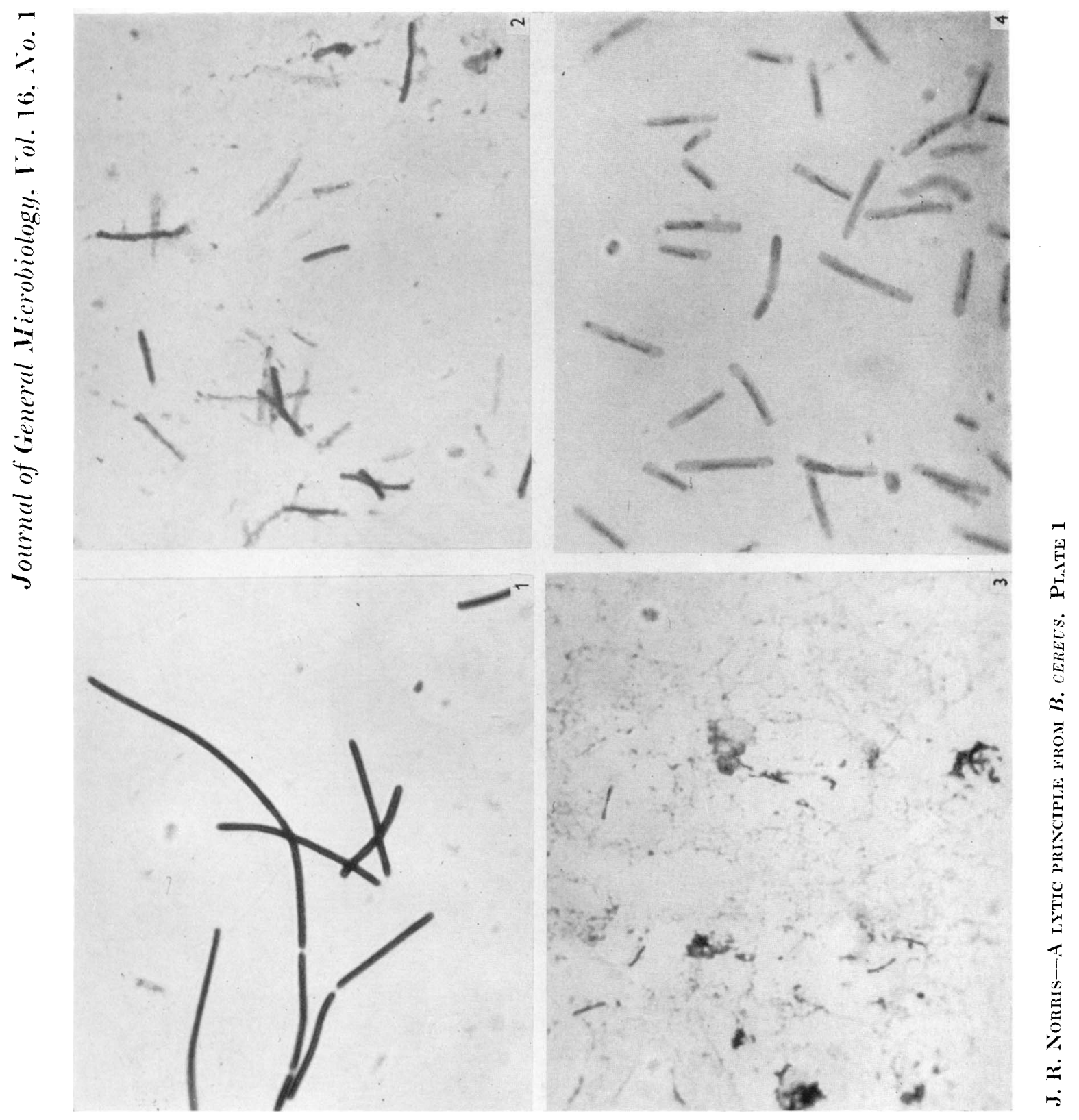

(Facing $p .8$ ) 
Journal of General Microbiology, Vol. 16, No. 1
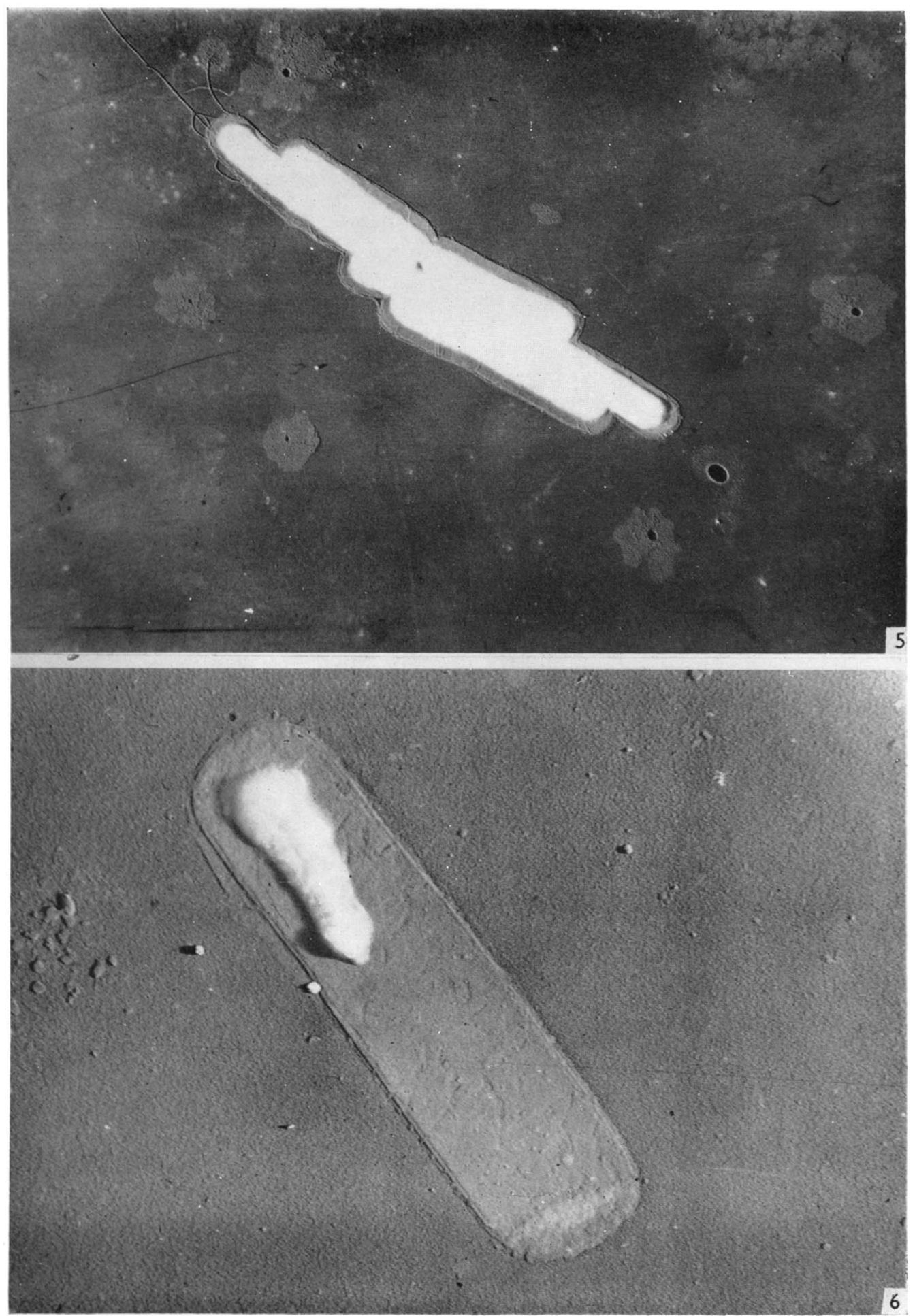

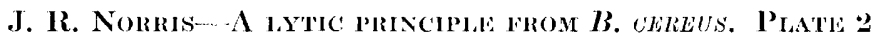

